Research Paper

\title{
Adenosquamous Carcinoma of the Stomach: A Population-based Study from the SEER Database
}

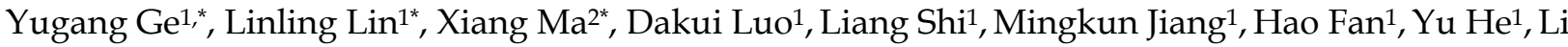 \\ Yang1,3 ${ }^{\bowtie}$, Zekuan $\mathrm{Xu}^{1}$ \\ 1. Department of General Surgery, The First Affiliated Hospital of Nanjing Medical University, Nanjing, Jiangsu Province, China \\ 2. Department of General Surgery, The Second Affiliated Hospital of Nanjing Medical University, Nanjing, Jiangsu, China \\ 3. Department of General Surgery, Liyang People's Hospital, Liyang Branch Hospital of Jiangsu Province Hospital, Liyang, Jiangsu Province, China \\ *Yugang Ge, Linling Lin, and Xiang Ma contributed equally to this work.
}

$\triangle$ Corresponding author: Li Yang, Department of General Surgery, The First Affiliated Hospital of Nanjing Medical University, No 300 Guangzhou Road, Nanjing 210000, Jiangsu Province, China; Department of General Surgery, Liyang People's Hospital, Liyang Branch Hospital of Jiangsu Province Hospital, Liyang, Jiangsu Province, China. Tel: +8613813998039, Email: pwkyangli@njmu.edu.cn

(C) The author(s). This is an open access article distributed under the terms of the Creative Commons Attribution License (https://creativecommons.org/licenses/by/4.0/). See http://ivyspring.com/terms for full terms and conditions.

Received: 2018.08.11; Accepted: 2019.06.22; Published: 2019.09.07

\begin{abstract}
Purpose: Gastric adenosquamous carcinoma (ASC) is a rare pathological type with poorly understood clinicopathological features. The purpose of this study is to identify the characteristics of gastric ASC patients.

Methods: Using the Surveillance, Epidemiology, and End Results (SEER) database (2000 to 2014), patients with ASC $(N=93)$ or adenocarcinoma $(A C)(N=41794)$ of the stomach were included. The epidemiology, tumor features, treatment, and outcomes between these two groups were compared.

Results: The incidences of ASC from 1983 to 2014 [annual percentage change (APC) $=-3.5 \%, 95 \%$ confidence interval $(\mathrm{Cl})-4.9$ to -2.1 ] and $\mathrm{AC}$ from 1973-2014 [APC $=-1.8 \%, 95 \% \mathrm{Cl}-2.0$ to -1.6 ] decreased over time. Compared to AC cases, patients with ASC were more likely to present poor differentiation (74.2\% vs $52.4 \%$ ) and later summary stage (distant: $46.2 \%$ vs $33.6 \%$ ) or later T stage (T4: $15.1 \%$ vs $9.0 \%$ ). Besides, the proportion of patients with distant metastasis (33.3\% vs $23.9 \%$ ), and chemotherapy (44.1\% vs $34.0 \%$ ) in ASC group was higher. The Kaplan-Meier analyses showed ASC cases had worse overall survival (OS) $(p=0.017)$ than that of $A C$ after propensity score matching (PSM), but not the cancer-specific survival (CSS) $(p=0.849)$. The further subgroup analyses suggested no statistical significance between gastric ASC patients and AC patients for CSS. The multivariate cox proportional hazard analyses indicated that patients with distant summary stage $(H R=2.11, p=0.014)$, no surgery $(H R=2.22, p=0.016)$, and no/unknown chemotherapy $(H R=3.33$, $p<0.001$ ) were associated with poor OS for ASC population alone. However, for CSS, only ASC cases with no/unknown chemotherapy $(\mathrm{HR}=2.22, p=0.018)$ indicated worse outcomes.

Conclusions: Gastric ASC presented more aggressive clinicopathologic characteristics and poorer OS compared with AC. The localized/regional summary stages and undergoing surgery suggested favorable OS for gastric ASC patients. ASC cases receiving chemotherary showed both better OS and CSS.
\end{abstract}

Key words: adenosquamous carcinoma, stomach, prognosis, SEER database

\section{Introduction}

In all pathologic types of gastric cancer, adenocarcinoma $(\mathrm{AC})$ is the most common, whereas the incidence of adenosquamous carcinoma (ASC) is extremely low, accounting for less than $1 \%$ of total gastric malignancies [1, 2]. ASC is a mixed neoplasia composed of AC and squamous cell carcinoma (SCC) 
components, with the latter making up at least $25 \%$ of the tumor mass [3]. Some previous studies indicated that its biological behaviors were generally determined by the AC component, but the results remained controversial [4-6].

Compared with the traditional AC, gastric ASC usually had aggressive clinicopathologic characteristics, such as larger tumor size, deeper tumor depth, lymph node invasion, and poorer survival $[7,8]$. However, due to its rarity, most ASC information was based on case reports or small single-institution studies, so that these results were not very convincing. Therefore, this study was conducted to describe the epidemiology, tumor features, treatments, and outcomes between patients with ASC and AC via Surveillance, Epidemiology, and End Results (SEER) database. In addition, the risk factors influencing the prognosis of gastric ASC patients were analyzed.

\section{Material and Methods}

\section{Study population}

Supported by the National Cancer Institute, the SEER program gathers information of 18 populationbased registered cancer institutes, which covers around $28 \%$ of the US population. According to the International Classification of Disease for Oncology, 3rd edition (ICD-0-3), we selected patients with gastric AC (SEER codes 8140-8145, 8210, 8211, 8214, $8220,8221,8255,8260-8263,8310,8480,8481,8570$, and 8574-8576) [9] and ASC (SEER code 8560) [10] during 2000 and 2014. All patients' data regarding the age, gender, race, marital status, primary site, grade, summary stage, $\mathrm{T}$ stage, lymph node metastasis, distant metastasis, surgery, radiation, and chemotherapy were extracted from the database. The endpoints were overall survival (OS) and cancer-specific survival (CSS). OS was defined as the time interval from diagnosis to death due to any cause, and CSS was the follow-up time from diagnosis to death due to gastric cancer. No personal identifying data were obtained from the SEER database. This study was approved by the review board at the First Affiliated Hospital of Nanjing Medical University.

\section{Statistical analysis}

The SEER*Stat software, version 8.3.5 was used to estimate the age-adjusted incidence as diagnoses per 100, 000 patients per year. The Joinpoint software, version 4.6.0 was performed to calculate the annual percentage changes (APCs) for assessing the changes of incidence. The GraphPad Prism 6 software was adopted to draw the figure of incidence. The Chi-square test was conducted to compare the categorical variables. The Student's t-test was used to compare the continuous variables satisfying the normal distribution and homogeneity of variance, otherwise, the Mann-Whitney U test was performed. The Kaplan-Meier method and log-rank test were used to draw survival curves and evaluate the differences of OS and CSS between gastric ASC and AC patients, or for ASC patients alone by each covariate. The univariate and multivariable Cox proportional hazards models were carried out to determine the independent prognostic factors and the results were exhibited by the hazard ratio (HR) and 95\% confidence interval (95\% CI). We generated the 1:1 matched ASC group and AC group via a propensity score matching (PSM) method, reducing the effects of differences in baseline features. The characteristics for matching ASC and AC were age, race, grade, summary stage, $\mathrm{T}$ stage, distant metastasis, surgery, and chemotherapy. The statistical analyses were completed by SPSS 22.0, and R softwares. The $p$ value $<0.05$ was considered to be significant.

\section{Results}

\section{Population features}

We selected gastric ASC and AC patients from the SEER database during 1973 and 2014 to describe the incidence of these two histological types. Because the Joinpoint software cannot process records with dependent variable $=0$ (the incidence of ASC at 1982 is 0), we calculated the APCs of ASC from 1973 to 1981 and from 1983 to 2014 . The results showed that the age-adjusted incidences of ASC (1983-2014) and AC (1973-2014) significantly decreased over time $(p<0.05)$, with the APCs of $-3.5 \%$ [95\% CI -4.9 to -2.1$]$ and $-1.8 \%$ [95\% CI -2.0 to -1.6$]$, respectively. However, there was no distinct decreased trend for the incidence of ASC during 1973 and 1981 (Figure 1).

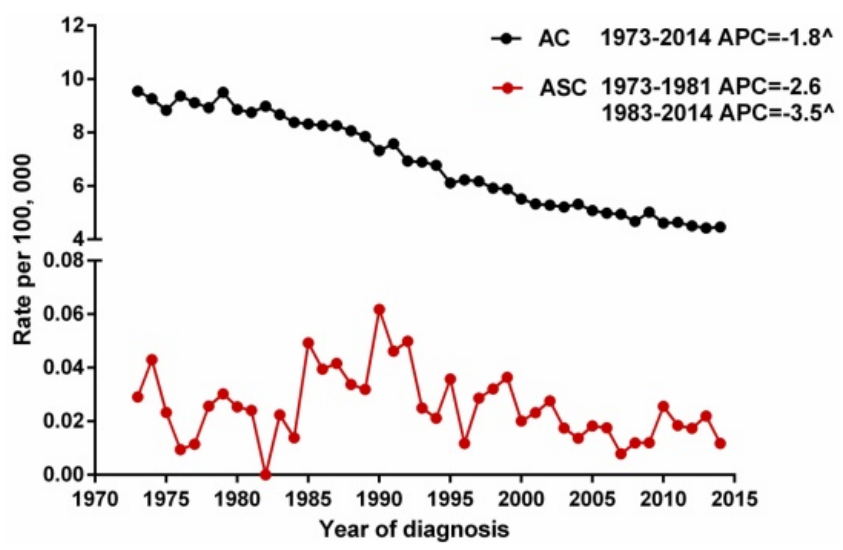

Figure 1. The age-adjusted incidences of gastric ASC and AC patients between 1973 and 2014 from the SEER database. ^indicated that the APC is significantly different from zero at the alpha $=0.05$ level. ASC: adenosquamous carcinoma; AC: adenocarcinoma; APC: annual percent change. 
Table 1. Characteristics of patients with gastric ASC and AC

\begin{tabular}{|c|c|c|c|}
\hline Characteristics & ASC & AC & $p$ value \\
\hline Number & $93(0.2 \%)$ & $41794(99.8 \%)$ & \\
\hline Age (years) & $68.72 \pm 12.42$ & $70.85 \pm 15.19$ & 0.078 \\
\hline \multicolumn{4}{|l|}{ Gender } \\
\hline Female & $35(37.6 \%)$ & $17194(41.1 \%)$ & 0.528 \\
\hline Male & $58(62.4 \%)$ & $24600(58.9 \%)$ & \\
\hline \multicolumn{4}{|l|}{ Ethnicity } \\
\hline White & $56(60.2 \%)$ & $26146(62.6 \%)$ & 0.078 \\
\hline Black & $25(26.9 \%)$ & $7280(17.4 \%)$ & \\
\hline Other & $12(12.9 \%)$ & $8262(19.8 \%)$ & \\
\hline Unknown & 0 & $106(0.3 \%)$ & \\
\hline \multicolumn{4}{|l|}{ Marital status } \\
\hline Single & $14(15.1 \%)$ & $5222(12.5 \%)$ & 0.128 \\
\hline Married & $58(62.4 \%)$ & $22164(53.0 \%)$ & \\
\hline Separated/divorced & $6(6.5 \%)$ & $3402(8.1 \%)$ & \\
\hline Widowed & $10(10.8 \%)$ & $8837(21.1 \%)$ & \\
\hline Other/unknown & $5(5.4 \%)$ & $2169(5.2 \%)$ & \\
\hline \multicolumn{4}{|l|}{ Primary site } \\
\hline Fundus & $9(9.7 \%)$ & $2403(5.7 \%)$ & 0.155 \\
\hline Body & $8(8.6 \%)$ & $5244(12.5 \%)$ & \\
\hline Antrum & $26(28.0 \%)$ & $12233(29.3 \%)$ & \\
\hline Pylorus & $7(7.5 \%)$ & $1871(4.5 \%)$ & \\
\hline Lesser curvature, NOS & $14(15.1 \%)$ & $4884(11.7 \%)$ & \\
\hline Greater curvature, NOS & $4(4.3 \%)$ & $2197(5.3 \%)$ & \\
\hline Overlapping lesion & $13(14.0 \%)$ & $4304(10.3 \%)$ & \\
\hline Stomach, NOS & $12(12.9 \%)$ & $8658(20.7 \%)$ & \\
\hline \multicolumn{4}{|c|}{ Pathological differentiation } \\
\hline Well & $1(1.1 \%)$ & $1924(4.6 \%)$ & 0.002 \\
\hline Moderate & $11(11.8 \%)$ & $11031(26.4 \%)$ & \\
\hline Poor & $69(74.2 \%)$ & $21885(52.4 \%)$ & \\
\hline Undifferentiated & $2(2.2 \%)$ & $637(1.5 \%)$ & \\
\hline Unknown & $10(10.8 \%)$ & $6317(15.1 \%)$ & \\
\hline \multicolumn{4}{|l|}{ Summary stage } \\
\hline In situ & 0 & $555(1.3 \%)$ & 0.002 \\
\hline Localized & $9(9.7 \%)$ & $10009(23.9 \%)$ & \\
\hline Regional & $37(39.8 \%)$ & $12785(30.6 \%)$ & \\
\hline Distant & $43(46.2 \%)$ & $14026(33.6 \%)$ & \\
\hline Unstaged & $4(4.3 \%)$ & $4419(10.6 \%)$ & \\
\hline \multicolumn{4}{|l|}{ T stage } \\
\hline $\mathrm{T} 0 / \mathrm{Tis} / \mathrm{T} 1$ & $7(7.5 \%)$ & $7740(18.5 \%)$ & 0.036 \\
\hline $\mathrm{T} 2$ & $19(20.4 \%)$ & $8132(19.5 \%)$ & \\
\hline $\mathrm{T} 3$ & $9(9.7 \%)$ & $3428(8.2 \%)$ & \\
\hline $\mathrm{T} 4$ & $14(15.1 \%)$ & $3762(9.0 \%)$ & \\
\hline Unknown & $44(47.3 \%)$ & $18732(44.8 \%)$ & \\
\hline \multicolumn{4}{|l|}{ Lymph node metastasis } \\
\hline Yes & $32(34.4 \%)$ & $11747(28.1 \%)$ & 0.259 \\
\hline No & $23(24.7 \%)$ & $13233(31.7 \%)$ & \\
\hline Unknown & $38(40.9 \%)$ & $16814(40.2 \%)$ & \\
\hline \multicolumn{4}{|l|}{ Distant metastasis } \\
\hline Yes & $31(33.3 \%)$ & $9970(23.9 \%)$ & 0.026 \\
\hline No & $28(30.1 \%)$ & $17871(42.8 \%)$ & \\
\hline Unknown & $34(36.6 \%)$ & $13953(33.4 \%)$ & \\
\hline \multicolumn{4}{|l|}{ Surgery } \\
\hline Yes & $57(61.3 \%)$ & $21113(50.5 \%)$ & 0.080 \\
\hline No & $36(38.7 \%)$ & $20130(48.2 \%)$ & \\
\hline Unknown & 0 & $551(1.3 \%)$ & \\
\hline \multicolumn{4}{|l|}{ Radiation } \\
\hline Yes & $19(20.4 \%)$ & $6880(16.5 \%)$ & 0.326 \\
\hline No/unknown & $74(79.6 \%)$ & $34914(83.5 \%)$ & \\
\hline \multicolumn{4}{|l|}{ Chemotherapy } \\
\hline Yes & $41(44.1 \%)$ & $14218(34.0 \%)$ & 0.048 \\
\hline No/unknown & $52(55.9 \%)$ & $27576(66.0 \%)$ & \\
\hline
\end{tabular}

Given that the treatment modalities, perioperative care, surgical methods and devices, and even the diagnostic ability change a lot, which will cause bias and affect the results. So, we extracted the data of ASC and AC patients between 2000 and 2014 from the SEER database. As a result, a total of 41887 gastric cancer patients including 93 ASC cases and 41794 AC cases were identified. The clinical characteristics of these subjects were summarized in Table 1. No remarkable difference was found between the two groups in terms of age, gender, ethnicity, marital status, primary site, lymph node metastasis, surgery, and radiation. ASC tumors tended to present a later summary stage (Distant: $46.2 \%$ vs $33.6 \%$, $p=0.002$ ), a later T stage (T4: $15.1 \%$ vs $9.0 \%, p=0.036$ ), and a higher distant metastasis rate $(33.3 \%$ vs $23.9 \%$, $p=0.026)$ when compared to those with AC. Also, the ASC group was obviously correlative with a higher tumor grade (poor: $74.2 \%$ vs $52.4 \%$; undifferentiated: $2.2 \%$ vs $1.5 \%, p=0.002$ ) and more likely to receive chemotherapy $(44.1 \%$ vs $34.0 \%, p=0.048)$.

\section{OS and CSS analyses}

We used the Kaplan-Meier method to evaluate OS and CSS among gastric ASC and AC patients, excluding the cases with unknown survival time. As showed in Figure 2A, patients with ASC showed worse OS than AC patients (1-, 3-, and 5-year OS: $28.0 \%$ vs $42.6 \%, 9.7 \%$ vs $20.3 \%, 5.4 \%$ vs $12.9 \%$, respectively, $p<0.01)$. In the ASC group alone, compared to the cases with localized or regional disease, patients in distant summary stage didn't survive more than 2 years, having poorer OS ( $p=0.042$ and $p<0.001$, respectively, Figure $2 \mathrm{~B}$ ). Interestingly, lymph node metastasis was significantly correlated to better OS $(p<0.001$, Figure $2 C)$, however, ASC patients with distant metastasis remained worse OS $(p<0.001$, Figure 2D). Figure 2E exhibited that single ASC patients had a shorter OS than married $(p=0.030)$. Furthermore, surgery, radiation, and chemotherapy could improve OS of ASC patients $(p=0.001, p<0.001$, and $p=0.002$, respectively, Figure $2 \mathrm{~F}-\mathrm{H})$.

For CSS analysis, unexpectedly, there was no statistical difference between ASC patients and AC patients $(p=0.930$, Figure 3A). Similarly, in the ASC group alone, patients with distant summary stage presented worse CSS than those with regional disease $(p=0.004$, Figure 3B). The influences of marital status on CSS were consistent with the results of OS, that is, single ASC patients had shorter CSS than married $(p=0.022$, Figure $3 C)$. Also, surgery, radiation, and chemotherapy could prolong CSS of ASC cases ( $p=0.007, p=0.004$, and $p=0.014$, respectively, Figure 3D-F).

Then, the prognostic factors associated with OS and CSS were further identified via univariate and multivariate Cox proportional hazard models in the ASC group. Table 2 summarized that distant summary stage and distant metastasis were significantly associated with poorer OS $(p<0.05)$. Conversely, surgery, radiation, and chemotherapy 
were protective factors for OS $(p<0.01$ for all). The results of multivariate analyses demonstrated that distant summary stage $(\mathrm{HR}=2.11, p=0.014)$, surgery $(\mathrm{HR}=0.45, p=0.016)$, and chemotherapy $(\mathrm{HR}=0.30$, $p<0.001)$ remained independent risk factors for OS in gastric ASC patients. Interestingly, the lymph node metastasis was found to be a protective factor in univariate Cox analysis, however, there was no significant difference after multivariate analysis

A

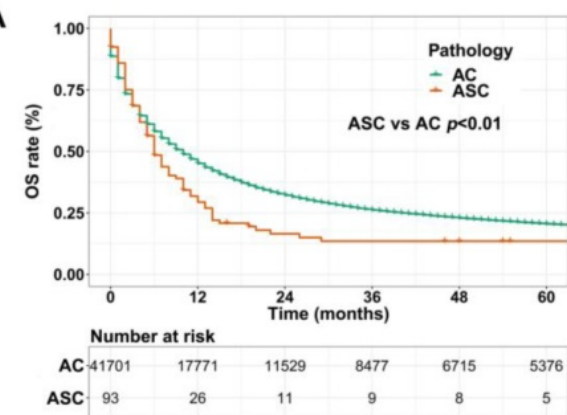

C

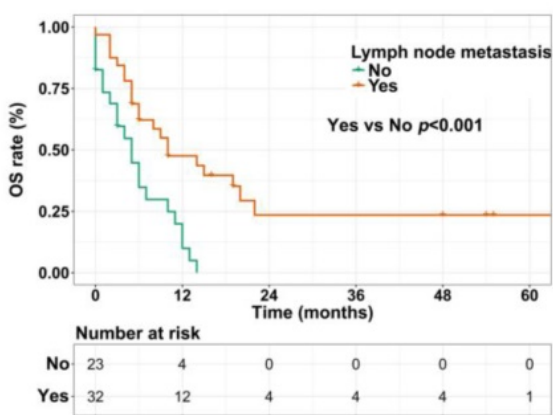

E

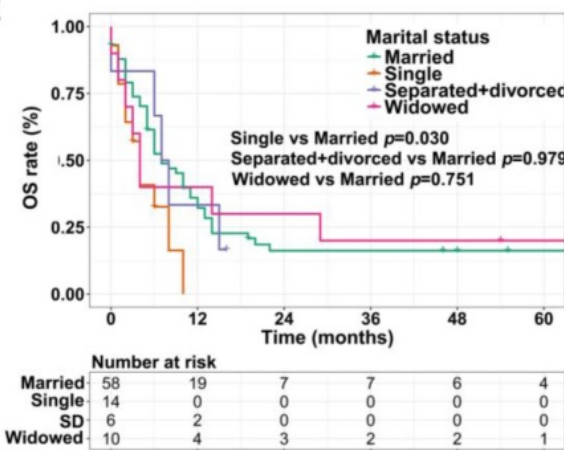

G

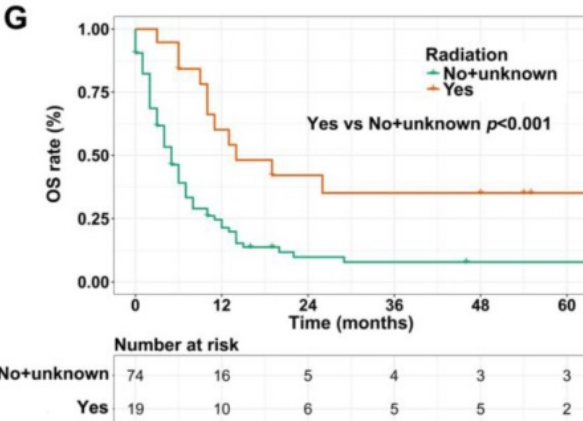

$(p=0.054)$. For CSS analyses, Table 3 showed that ASC patients at distant summary stage had worse CSS in univariate analysis $(p=0.008)$. And the CSS of ASC cases receiving surgery $(p=0.012)$ and radiation $(p=0.009)$, and chemotherapy $(p=0.022)$ was longer. We found that chemotherapy (HR=0.45, $p=0.018$ ) could impact the CSS independently through multivariate analyses.
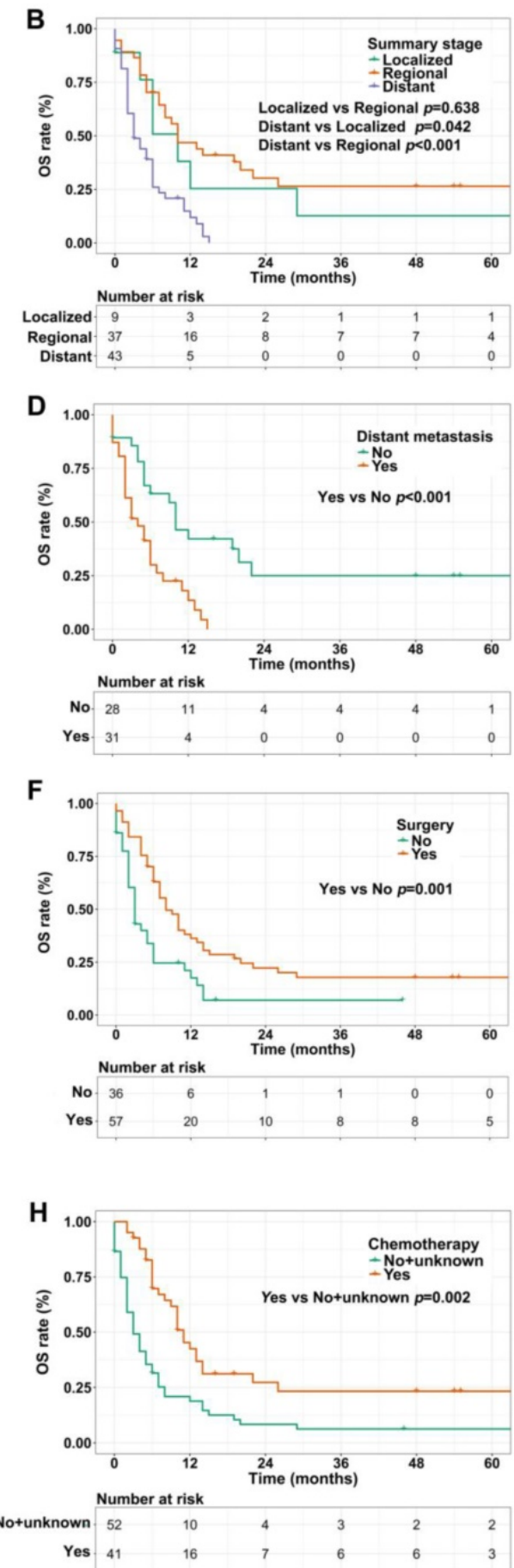

Figure 2. (A) OS for patients with gastric ASC and AC; (B) OS for ASC patients with distant, regional, and localized stages; (C) OS for ASC patients with lymph node metastasis or not; (D) OS for ASC patients with distant metastasis or not; (E) OS for ASC patients with different marital statuses; (F) OS for ASC patients receiving surgery or not; (G) OS for ASC patients receiving radiation or no/unknown; (H) OS for ASC patients receiving chemotherapy or no/unknown. ASC: adenosquamous carcinoma; AC: adenocarcinoma; OS: overall survival; SD: separated/divorced. 


\section{Survival analyses in matched groups}

We performed 1:1 matched analysis by PSM to match ASC patients with AC patients, balancing the differences of the baseline characteristics. A total of 186 gastric cancer patients consisting of 93 ASC cases and $93 \mathrm{AC}$ cases were obtained after PSM and no significant differences were observed in basic clinical features ( $p>0.05$ for all) (Table 4). Using the Kaplan Meier method, we still found that matched ASC patients had worse OS $(p=0.017)$, but not CSS $(p=0.849)$ than matched AC (Figure 4A and B). The subgroup analyses were carried out to elucidate whether the CSS between the ASC and AC groups existed differences. Regrettably, the results of univariate Cox analyses suggested no statistical difference between ASC patients and AC patients in all subgroups ( $p>0.05$, Figure 5).

\section{Discussion}

The incidence of gastric ASC is extremely low, only comprising $<1 \%$ of all gastric malignancies. Most studies about gastric ASC were limited to case reports or small series, so our population-based study used data from SEER between 2000 and 2014 to better understand the clinicopathological features and prognosis of it. As expected, the results confirmed the rarity of gastric ASC (ASC: 0.2\%, AC: $99.8 \%$ ) again. It's worth noting that the incidences of ASC from 1983 to 2014 and AC from 1973-2014 decreased over time, which likely attributed to the prevention of $\mathrm{H}$. pylori colonization, reduced salt intake, increased screening rate of gastroscopy, and so on.
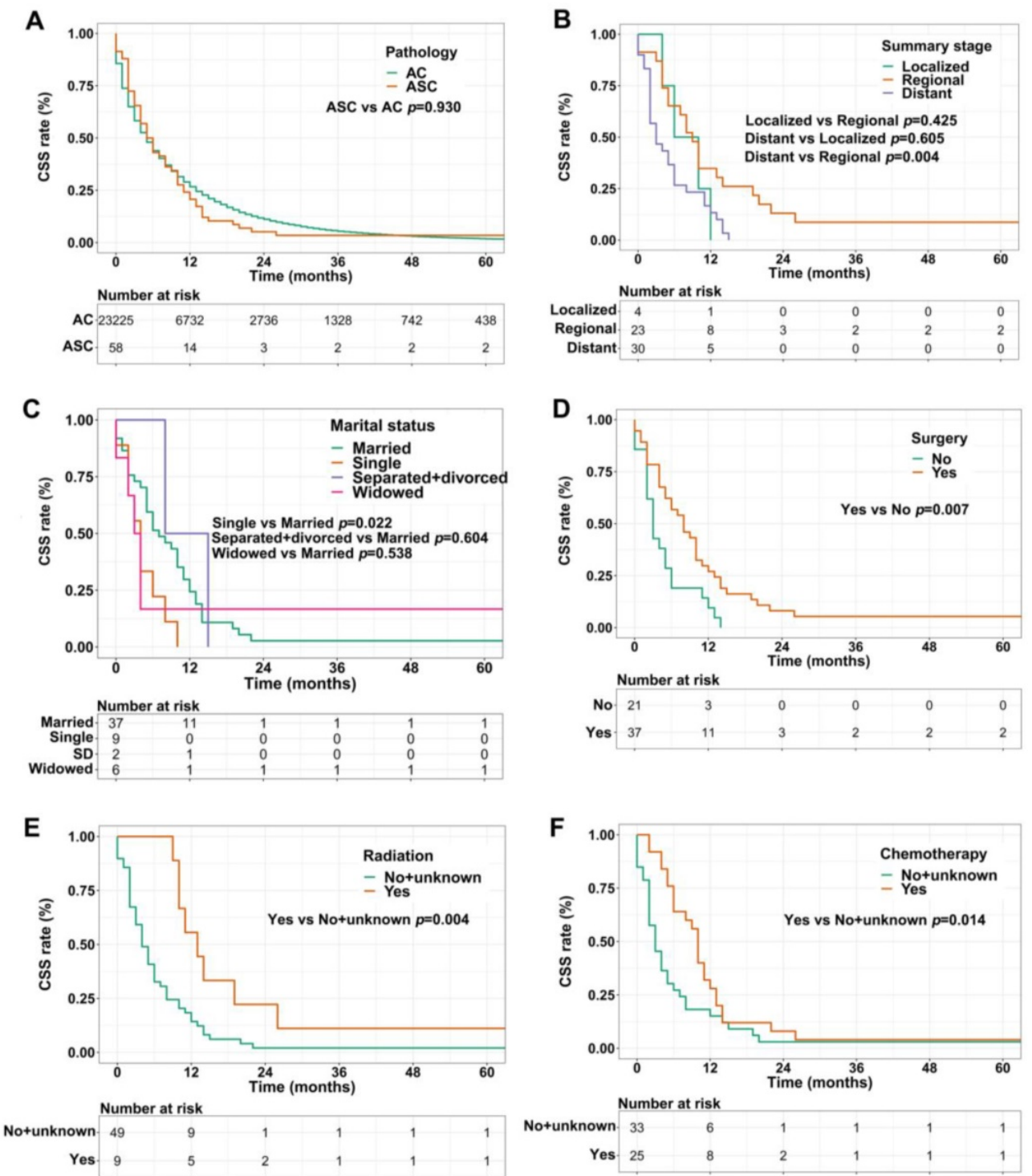

Figure 3. (A) CSS for patients with gastric ASC and AC; (B) CSS for ASC patients with distant, regional, and localized stages; (C) CSS for ASC patients with different marital statuses; (D) CSS for ASC patients receiving surgery or not; (E) CSS for ASC patients receiving radiation or no/unknown; (F) CSS for ASC patients receiving chemotherapy or no/unknown. ASC: adenosquamous carcinoma; AC: adenocarcinoma; CSS: cancer specific survival; SD: separated/divorced. 
A

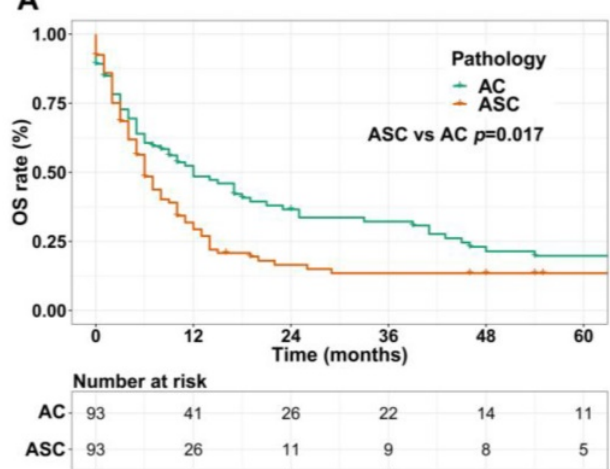

B

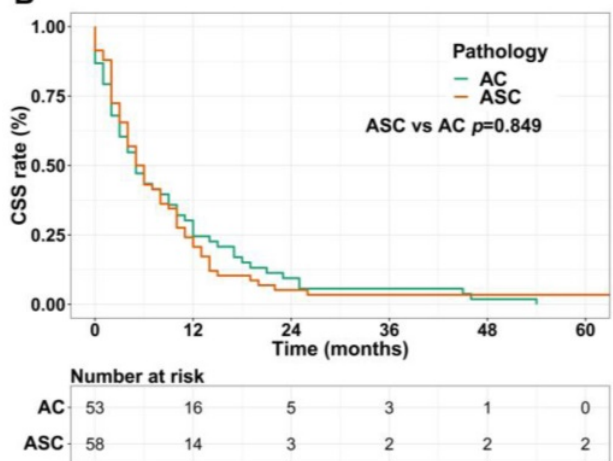

Figure 4. (A) OS for patients with gastric ASC and AC after PSM; (B) CSS for patients with gastric ASC and AC after PSM. ASC: adenosquamous carcinoma; AC: adenocarcinoma; PSM: propensity score matching.

Table 2. Univariate and Multivariate Cox proportional hazard analyses of clinical characteristics for OS in patients with gastric ASC

\begin{tabular}{|c|c|c|c|c|c|}
\hline \multirow[t]{2}{*}{ Factor } & \multirow[t]{2}{*}{ Category } & \multicolumn{2}{|l|}{ Univariate } & \multicolumn{2}{|l|}{ Multivariate } \\
\hline & & HR (95\% CI) & $p$ value & HR $(95 \%$ CI) & $p$ value \\
\hline Age & $>60$ vs $\leq 60$ & $1.24(0.70-2.17)$ & 0.466 & & \\
\hline Gender & Male vs Female & $0.95(0.60-1.52)$ & 0.841 & & \\
\hline Ethnicity & White vs Black/other & $0.74(0.46-1.17)$ & 0.198 & & \\
\hline Primary site & Antrum/pylorus vs Fundus/body & $0.56(0.30-1.04)$ & 0.067 & & \\
\hline Pathological differentiation & Poor/undifferentiated vs Well/moderate & $0.90(0.44-1.84)$ & 0.779 & & \\
\hline Summary stage & Distant vs Localized/regional & $2.90(1.76-4.78)$ & $<0.001$ & $2.11(1.17-3.83)$ & 0.014 \\
\hline T stage & $\mathrm{T} 3 / \mathrm{T} 4$ vs $\mathrm{T} 1 / \mathrm{T} 2$ & $0.89(0.47-1.68)$ & 0.718 & & \\
\hline Lymph node metastasis & Yes vs No & $0.39(0.21-0.72)$ & 0.003 & $0.49(0.24-1.01)$ & 0.054 \\
\hline Distant metastasis & Yes vs No & $2.69(1.46-4.94)$ & 0.001 & $1.69(0.82-3.47)$ & 0.156 \\
\hline \multirow[t]{3}{*}{ Marital status } & Single vs Married & $1.98(1.00-3.91)$ & 0.050 & & \\
\hline & Separated/divorced vs Married & $1.04(0.41-2.65)$ & 0.922 & & \\
\hline & Widowed vs Married & $1.10(0.54-2.26)$ & 0.794 & & \\
\hline Surgery & Yes vs No & $0.48(0.30-0.77)$ & 0.002 & $0.45(0.24-0.86)$ & 0.016 \\
\hline Radiation & Yes vs No/unknown & $0.38(0.20-0.70)$ & 0.002 & $0.78(0.40-1.52)$ & 0.471 \\
\hline Chemotherapy & Yes vs No/unknown & $0.42(0.27-0.67)$ & $<0.001$ & $0.30(0.17-0.52)$ & $<0.001$ \\
\hline
\end{tabular}

ASC: adenosquamous carcinoma; AC: adenocarcinoma; OS: overall survival. The significant $p$ values are in bold.

Table 3. Univariate and Multivariate Cox proportional hazard analyses of clinical characteristics for CSS in patients with gastric ASC

\begin{tabular}{|c|c|c|c|c|c|}
\hline \multirow[t]{2}{*}{ Factor } & \multirow[t]{2}{*}{ Category } & \multicolumn{2}{|l|}{ Univariate } & \multicolumn{2}{|l|}{ Multivariate } \\
\hline & & HR $(95 \%$ CI) & $p$ value & HR $(95 \%$ CI) & $p$ value \\
\hline Age & $>60$ vs $\leq 60$ & $1.05(0.54-2.04)$ & 0.887 & & \\
\hline Gender & Male vs Female & $0.99(0.58-1.69)$ & 0.979 & & \\
\hline Ethnicity & White vs Black/other & $0.89(0.52-1.51)$ & 0.658 & & \\
\hline Primary site & Antrum/pylorus vs Fundus/Body & $1.00(0.49-2.05)$ & 0.995 & & \\
\hline Pathological differentiation & Poor/undifferentiated vs Well/moderate & $0.55(0.24-1.27)$ & 0.160 & & \\
\hline Summary stage & Distant vs Localized/regional & $2.16(1.23-3.81)$ & 0.008 & $1.53(0.73-3.22)$ & 0.262 \\
\hline T stage & $\mathrm{T} 3 / \mathrm{T} 4$ vs $\mathrm{T} 1 / \mathrm{T} 2$ & $1.00(0.48-2.05)$ & 0.989 & & \\
\hline Lymph node metastasis & Yes vs No & $0.70(0.34-1.45)$ & 0.335 & & \\
\hline Distant metastasis & Yes vs No & $1.58(0.80-3.10)$ & 0.186 & & \\
\hline \multirow[t]{3}{*}{ Marital status } & Single vs Married & $2.03(0.95-4.34)$ & 0.067 & & \\
\hline & Separated/divorced vs Married & $0.72(0.17-2.99)$ & 0.647 & & \\
\hline & Widowed vs Married & $1.24(0.50-3.05)$ & 0.641 & & \\
\hline Surgery & Yes vs No & $0.48(0.27-0.85)$ & 0.012 & $0.50(0.23-1.12)$ & 0.091 \\
\hline Radiation & Yes vs No/unknown & $0.36(0.17-0.78)$ & 0.009 & $0.66(0.28-1.58)$ & 0.352 \\
\hline Chemotherapy & Yes vs No/unknown & $0.54(0.31-0.91)$ & 0.022 & $0.45(0.23-0.87)$ & 0.018 \\
\hline
\end{tabular}

ASC: adenosquamous carcinoma; AC: adenocarcinoma; CSS: cancer specific survival. The significant $p$ values are in bold.

In the present study, the average age of patients with gastric ASC was 68.7 years (range: 29 to 89 years), which was older than Feng et al.'s report with a mean age of 61.3 years [8]. With regard to sex, our study indicated that the male to female ratio was 1.66, agreeing with Chen et al.'s result (3.3:1) and suggesting more males could be affected than females [6]. In term of ethnicity, most gastric ASC patients belonged to the white race $(60.2 \%)$, this was likely owing to the race distribution of western population. As for the primary site, we took Honda et al.'s study as reference [9], treating "antrum/pylorus" as synonymous with the lower third. Our results showed that the most common location of ASC was antrum/pylorus (35.5\%), consistent with Feng et al. and Ajoodhea et al.'s reports [10]. Although other 
researches revealed that lesions of most gastric ASC were located at the upper third of stomach $[6,11,12]$, but they included few patients and lacked enough persuasion.

Table 4. Characteristics of patients with gastric ASC and AC after PSM

\begin{tabular}{|c|c|c|c|}
\hline Characteristics & ASC & $\mathrm{AC}$ & $p$ value \\
\hline Number & 93 & 93 & \\
\hline Age (years) & $68.72 \pm 12.42$ & $69.23 \pm 13.70$ & 0.825 \\
\hline \multicolumn{4}{|l|}{ Gender } \\
\hline Female & $35(37.6 \%)$ & $36(38.7 \%)$ & 1.000 \\
\hline Male & $58(62.4 \%)$ & $57(61.3 \%)$ & \\
\hline \multicolumn{4}{|l|}{ Ethnicity } \\
\hline White & $56(60.2 \%)$ & $59(63.4 \%)$ & 0.868 \\
\hline Black & $25(26.9 \%)$ & $22(23.7 \%)$ & \\
\hline Other & $12(12.9 \%)$ & $12(12.9 \%)$ & \\
\hline \multicolumn{4}{|l|}{ Marital status } \\
\hline Single & $14(15.1 \%)$ & $14(15.1 \%)$ & 0.779 \\
\hline Married & $58(62.4 \%)$ & $51(54.8 \%)$ & \\
\hline Separated/divorced & $6(6.5 \%)$ & $10(10.8 \%)$ & \\
\hline Widowed & $10(10.8 \%)$ & $13(14.0 \%)$ & \\
\hline Other/unknown & $5(5.4 \%)$ & $5(5.4 \%)$ & \\
\hline \multicolumn{4}{|l|}{ Primary site } \\
\hline Fundus & $9(9.7 \%)$ & $9(9.7 \%)$ & 0.580 \\
\hline Body & $8(8.6 \%)$ & $8(8.6 \%)$ & \\
\hline Antrum & $26(28.0 \%)$ & $30(32.3 \%)$ & \\
\hline Pylorus & $7(7.5 \%)$ & $2(2.2 \% 0$ & \\
\hline Lesser curvature, NOS & $14(15.1 \%)$ & $11(11.8 \%)$ & \\
\hline Greater curvature, NOS & $4(4.3 \%)$ & $6(6.5 \%)$ & \\
\hline Overlapping lesion & $13(14.0 \%)$ & $9(9.7 \%)$ & \\
\hline Stomach, NOS & $12(12.9 \%)$ & $18(19.4 \%)$ & \\
\hline \multicolumn{4}{|c|}{ Pathological differentiation } \\
\hline Well & $1(1.1 \%)$ & $2(2.2 \%)$ & 0.263 \\
\hline Moderate & $11(11.8 \%)$ & $14(15.1 \%)$ & \\
\hline Poor & $69(74.2 \%)$ & $57(61.3 \%)$ & \\
\hline Undifferentiated & $2(2.2 \%)$ & $1(1.1 \%)$ & \\
\hline Unknown & $10(10.8 \%)$ & $19(20.4 \%)$ & \\
\hline \multicolumn{4}{|l|}{ Summary stage } \\
\hline In situ & 0 & $1(1.1 \%)$ & 0.746 \\
\hline Localized & $9(9.7 \%)$ & $11(11.8 \%)$ & \\
\hline Regional & $37(39.8 \%)$ & $35(37.6 \%)$ & \\
\hline Distant & $43(46.2 \%)$ & $39(41.9 \%)$ & \\
\hline Unstaged & $4(4.3 \%)$ & $7(7.5 \%)$ & \\
\hline \multicolumn{4}{|l|}{ T stage } \\
\hline $\mathrm{T} 1$ & $7(7.5 \%)$ & $12(12.9 \%)$ & 0.663 \\
\hline $\mathrm{T} 2$ & $19(20.4 \%)$ & $16(17.2 \%)$ & \\
\hline T3 & $9(9.7 \%)$ & $8(8.6 \%)$ & \\
\hline $\mathrm{T} 4$ & $14(15.1 \%)$ & $10(10.8 \%)$ & \\
\hline Unknown & $44(47.3 \%)$ & $47(50.5 \%)$ & \\
\hline \multicolumn{4}{|l|}{ Lymph node metastasis } \\
\hline Yes & $32(34.4 \%)$ & $27(29.0 \%)$ & 0.693 \\
\hline No & $23(24.7 \%)$ & $23(24.7 \%)$ & \\
\hline Unknown & $38(40.9 \%)$ & $43(46.2 \%)$ & \\
\hline \multicolumn{4}{|l|}{ Distant metastasis } \\
\hline Yes & $31(33.3 \%)$ & $31(33.3 \%)$ & 1.000 \\
\hline No & $28(30.1 \%)$ & $28(30.1 \%)$ & \\
\hline Unknown & $34(36.6 \%)$ & $34(36.6 \%)$ & \\
\hline \multicolumn{4}{|l|}{ Surgery } \\
\hline Yes & $57(61.3 \%)$ & $54(58.1 \%)$ & 0.765 \\
\hline No & $36(38.7 \%)$ & $39(41.9 \%)$ & \\
\hline \multicolumn{4}{|l|}{ Radiation } \\
\hline Yes & $19(20.4 \%)$ & $22(23.7 \%)$ & 0.724 \\
\hline No/unknown & $74(79.6 \%)$ & $71(76.3 \%)$ & \\
\hline \multicolumn{4}{|l|}{ Chemotherapy } \\
\hline Yes & $41(44.1 \%)$ & $45(48.4 \%)$ & 0.659 \\
\hline No/unknown & $52(55.9 \%)$ & $48(51.6 \%)$ & \\
\hline
\end{tabular}

ASC: adenosquamous carcinoma; AC: adenocarcinoma; PSM: propensity score matching.
According to previous studies' results, gastric ASC, similar to the ASC occurring in other digestive system (such as esophagus, pancreas, and colon/rectum) [13-15], was extremely aggressive than AC. Our study also identified that patients with gastric ASC presented later summary stage, later $\mathrm{T}$ stage, and higher distant metastasis than those with AC. As we all known, gastric ASC was a mixed-pattern carcinoma of glandular and squamous components. Which kind of component mainly determined the malignant biological behavior of ASC, however, the results of previous studies remained inconsistent. Chen et al. [6] and Saito et al.'s [11] studies showed that both glandular and squamous components could lead to distant metastasis. Some other reports found that the biological behaviors of ASC were mainly determined by the AC component $[16,17]$. In all, the results based on single case report or small case series couldn't reach a convincing conclusion. Regrettably, our data from SEER database lacked the detail information about the component in metastatic lesions of ASC.

Due to the special etiology and biological features of ASC, gastric ASC patients carried a worse OS than $A C$ patients before and after 1:1 matching ASC with AC using PSM, which was in accordance with previous studies [7, 8]. In addition, this is the first study to evaluating the CSS between ASC patients and AC patients. However, for CSS analyses, whether we used PSM or not, the outcomes between these two pathology types showed no statistical differences. Therefore, we further performed the subgroup analyses and also found no statistical significance between ASC and AC patients in all subgroups. The possible explanation is that our study didn't had strong statistical power to detect the difference between ASC and AC cases due to too small sample size of ASC patients caused by the rarity of gastric ASC.

Then the risk factors for ASC patients' survival were analyzed, like Qin et al.' study [18], we included the SEER historic stage in the multivariate Cox analyses. The results revealed that distant summary stage was independently associated with poorer OS. As with gastric AC, tumor stage was the most significant prognostic factor. Yu et al.'s study observed that distal gastric cancer patients had a higher 5-year survival rate $(51 \%)$ than proximal gastric cancer (28\%) [19]. In our present research, however, patients with ASC located at antrum/pylorus sites didn't have better OS. Furthermore, previous studies have shown that diagnosis of cancer led to more distress than other diseases [20] and psychological stresses influenced immune function, contributing to tumor progression 


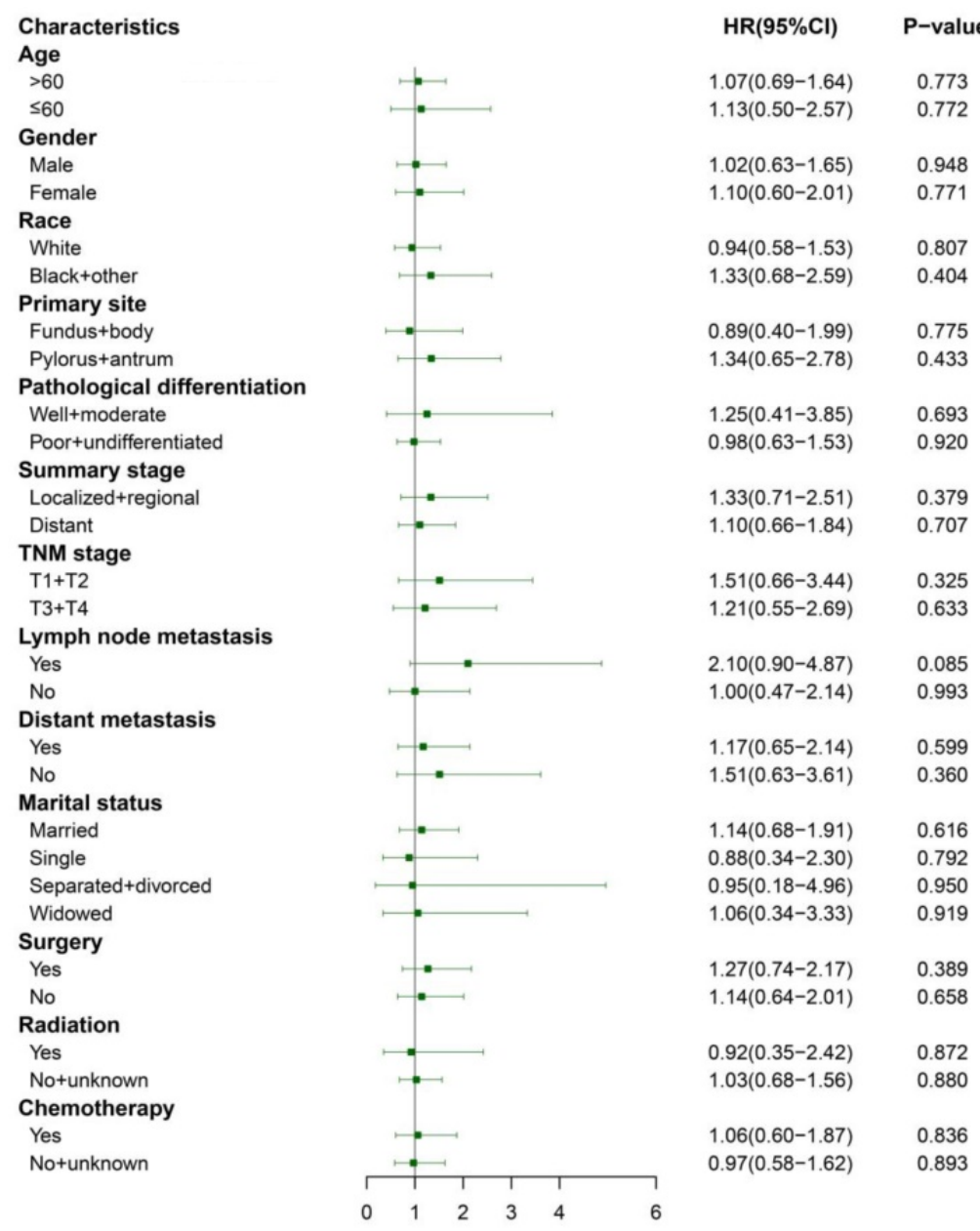

Figure 5. Subgroup analyses of CSS between the matched ASC and AC groups after PSM. ASC: adenosquamous carcinoma; AC: adenocarcinoma; PSM: propensity score matching. and mortality [21]. Married patients could obtain more social supports from their spouses or friends, in turn, displaying less distress and depression after the cancer diagnosis [22]. Our Kaplan-Meier analyses showed that compared with married patients, single patients had shorter OS. Nonetheless, no significant difference was found when we conducted the Cox univariate analysis, which was likely caused by low statistical power or the different biological behaviors of gastric ASC from traditional AC. Because of ASC's rarity, no special standard therapeutic strategies were well established. Few studies indicated that the chemotherapy with S-1 (plus paclitaxel) could improve survival $[1,23]$. In our study, a remarkably longer OS was also observed in patients receiving surgery and chemotherapy after multivariate analyses. Chen et al.'s study [6] reported that adjuvant radiotherapy could improve survival time due to the squamous components of ASC. Our results also showed the similar trend, but without a statistically significant difference. For CSS of gastric ASC patients, our study indicated only chemotherapy predicted better survival independently.
A few hypotheses have been proposed regarding the origin of SCC component in ASC [24]: (1) metaplastic transformation of AC; (2) cancerization of metaplastic squamous cells or ectopic squamous epithelium; (3) collision of AC and SCC; and (4) stem cell differentiation towards both glandular and squamous cells. However, the results remained controversial, and more studies are required to verify the histogenesis of gastric ASC.

Besides, similar with other retrospective studies using SEER as a data source, our study also had some limitations. Firstly, our results mainly illuminated the clinical features of ASC in California, not representing the patients in other areas. Secondly, we lacked clinicopathologic data (such as pathological differentiation, summary stage, T stage, lymph node metastases, and distant metastases) for some patients and didn't accurately describe the clinical parameters of gastric ASC. Thirdly, SEER database had no detailed chemotherapy regimens or information about targeted drugs, being not available to assess the impacts of specific treatment regimens. In the future studies, we will collect information of ASC patients from our own hospital, and further clarify the clinicopathologic characteristics of it.

\section{Conclusion}

Gastric ASC was a unique and rare histological type in gastric cancer. Compared with AC patients, ASC cases presented poorer differentiation, later summary stage, later $\mathrm{T}$ stage, a higher distant metastasis rate, and worse OS before and after PSM, but not for CSS. More ASC patients received chemotherapy. Multivariate analyses showed that early summary stage, surgery, and chemotherapy may be favorable for OS for patients with gastric ASC. For CSS, chemotherapy could improve the outcomes of ASC patients.

\section{Acknowledgments}

This work was supported by the Natural Science Foundation of Jiangsu Province (BK20171505), the National Natural Science Foundation of China (81874219), and the "Six One" Project of Jiangsu Province (IC17). 


\section{Competing Interests}

The authors have declared that no competing interest exists.

\section{References}

1. Ebi M, Shimura T, Yamada S, Hirata Y, Tsukamoto H, Okamoto Y, et al. A patient with gastric adenosquamous carcinoma with intraperitoneal free cancer cells who remained recurrence-free with postoperative S-1 chemotherapy. Intern Med. 2012; 51: 3125-9.

2. Faria GR, Eloy C, Preto JR, Costa EL, Almeida T, Barbosa J, et al. Primary gastric adenosquamous carcinoma in a Caucasian woman: a case report. J Med Case Rep. 2010; 4: 351.

3. Straus R, Heschel S, Fortmann DJ. Primary adenosquamous carcinoma of the stomach. A case report and review. Cancer. 1969; 24: 985-95.

4. Kim YS, Heo WS, Chae KH, Gang YS, Jung JH, Kim $\mathrm{SH}$, et al. [Clinicopathological features and differences of p53 and Ki-67 expression in adenosquamous and squamous cell carcinomas of the stomach]. Korean J Gastroenterol. 2006; 47: 425-31.

5. Bansal RK, Sharma P, Kaur R, Arora A. Primary gastric adenosquamous carcinoma in an Indian male. Indian J Pathol Microbiol. 2013; 56: 416-8.

6. Chen H, Shen C, Yin R, Yin Y, Chen J, Han L, et al. Clinicopathological characteristics, diagnosis, treatment, and outcomes of primary gastric adenosquamous carcinoma. World J Surg Oncol. 2015; 13: 136.

7. Mori M, Iwashita A, Enjoji M. Adenosquamous carcinoma of the stomach. A clinicopathologic analysis of 28 cases. Cancer. 1986; 57: 333-9.

8. Feng F, Zheng G, Qi J, Xu G, Wang F, Wang Q, et al. Clinicopathological features and prognosis of gastric adenosquamous carcinoma. Sci Rep. 2017; 7: 4597.

9. Honda M, Wong SL, Healy MA, Nakajima T, Watanabe M, Fukuma S, et al. Long-term Trends in Primary Sites of Gastric Adenocarcinoma in Japan and the United States. J Cancer. 2017; 8: 1935-42.

10. Ajoodhea H, Zhang RC, Xu XW, Jin WW, Chen K, He YT, et al. Fever as a first manifestation of advanced gastric adenosquamous carcinoma: a case report. World J Gastroenterol. 2014; 20: 10193-201.

11. Saito S, Hosoya Y, Morishima K, Ui T, Haruta H, Kurashina K, et al. A clinicopathological and immunohistochemical study of gastric cancer with squamous cell carcinoma components: a clinically aggressive tumor. J Dig Dis. 2012; 13: 407-13.

12. Quan J, Zhang R, Liang H, Li F, Liu H. The clinicopathologic and prognostic analysis of adenosquamous and squamous cell carcinoma of the stomach. Am Surg. 2013; 79: E206-8.

13. Yendamuri S, Malhotra U, Hennon M, Miller A, Groman A, Halloon A, et al. Clinical characteristics of adenosquamous esophageal carcinoma. J Gastrointest Oncol. 2017; 8: 89-95.

14. Boyd CA, Benarroch-Gampel J, Sheffield KM, Cooksley CD, Riall TS. 415 patients with adenosquamous carcinoma of the pancreas: a population-based analysis of prognosis and survival. J Surg Res. 2012; 174: 12-9.

15. Masoomi H, Ziogas A, Lin BS, Barleben A, Mills S, Stamos MJ, et al. Population-based evaluation of adenosquamous carcinoma of the colon and rectum. Dis Colon Rectum. 2012; 55: 509-14.

16. Lee WA, Woo DK, Kim YI, Kim WH. p53, p16 and RB expression in adenosquamous and squamous cell carcinomas of the stomach. Pathol Res Pract. 1999; 195: 747-52.

17. Chen YY, Li AF, Huang KH, Lan YT, Chen MH, Chao Y, et al. Adenosquamous carcinoma of the stomach and review of the literature. Pathol Oncol Res. 2015; 21: 547-51.

18. Qin BD, Jiao XD, Yuan LY, Liu K, Zang YS. Adenosquamous carcinoma of the bile duct: a population-based study. Cancer Manag Res. 2018; 10: 439-46.

19. Yu X, Hu F, Li C, Yao Q, Zhang H, Xue Y. Clinicopathologic characteristics and prognosis of proximal and distal gastric cancer. Onco Targets Ther. 2018; 11: 1037-44.

20. Kaiser NC, Hartoonian N, Owen JE. Toward a cancer-specific model of psychological distress: population data from the 2003-2005 National Health Interview Surveys. J Cancer Surviv. 2010; 4: 291-302.

21. Moreno-Smith $\mathrm{M}$, Lutgendorf SK, Sood AK. Impact of stress on cancer metastasis. Future Oncol. 2010; 6: 1863-81.

22. Goldzweig G, Andritsch E, Hubert A, Brenner B, Walach N, Perry S, et al Psychological distress among male patients and male spouses: what do oncologists need to know? Ann Oncol. 2010; 21: 877-83.

23. Hirano M, Ozamoto $Y$, Ichinose M, Togawa T, Takao N, Mizumoto A, et al. [A case of gastric adenosquamous carcinoma with peritoneal dissemination in which treatment with S-1 plus paclitaxel therapy resulted in improved ong-term survival]. Gan To Kagaku Ryoho. 2014; 41: 885-8.

24. Yoshida K, Manabe T, Tsunoda T, Kimoto M, Tadaoka Y, Shimizu M. Early gastric cancer of adenosquamous carcinoma type: report of a case and review of literature. Jpn J Clin Oncol. 1996; 26: 252-7. 